\title{
The screening and treatment of undiagnosed depression in patients with chronic obstructive pulmonary disease (COPD) in a general practice
}

\author{
*Kamran Ahmed ${ }^{a}$, Ashok Kelshikerb, Christopher Jenner \\ a SHO in Psychiatry, St. Mary's Psychiatric Training Scheme, London W10 6DZ. \\ ${ }^{b}$ General Practitioner, Elliott Hall Medical Centre, Hatchend, Pinner, Middlesex, HA5 4EA
}

Received 5th February 2007; accepted 12th April 2007

\begin{abstract}
Aims and Objectives: To screen for, and to treat, depression in COPD patients in a general practice surgery, and to test the feasibility of the screening method.

Methods: We sent The Beck Depression Inventory (BDI) Short-Form Questionnaire to all patients on the COPD register ( $n=69$ ) by post, excluding those already diagnosed with depression $(n=3)$. We sent appointments to all responders with moderate to severe depression and offered them treatment including antidepressants and pulmonary rehabilitation if the diagnosis was confirmed.

Results: Of the 66 patients to whom we sent questionnaires, 43 were returned completed and 13 of these had significant depression (30\%). 10 of these patients were contactable and attended for review. The diagnosis of depression was confirmed in all 10 cases. Treatment options were discussed; five chose antidepressant medication, three chose pulmonary rehabilitation and two declined treatment.

Conclusion: We identified and treated a significant number of depressed COPD patients in a general practice setting using this screening method. This method could have wider applications for screening, although further trials are needed.

(c) 2007 General Practice Airways Group. All rights reserved.

K Ahmed, et al. Prim Care Resp J 2007; 16(4): 249-251.

doi:10.3132/pcrj.2007.00048
\end{abstract}

Keyw ords COPD, depression, diagnosis, primary care, screening, treatment

\section{Introduction}

Studies have found that depression rates in patients with chronic obstructive pulmonary disease (COPD) range from 20$60 \%,{ }^{1}$ and one recent primary care study found a rate of $54 \%{ }^{2}$ Under-diagnosis of depression in COPD patients is thought to be a result of both doctor- and patient-related factors. ${ }^{3}$ Depression and anxiety predict health-related quality of life better than traditional lung function parameters. ${ }^{4}$ Once depression develops, patients are less adherent to treatment plans, suffer loss of function, and potentially suffer worse outcomes during and after exacerbations.' Furthermore, studies suggest that treatment of concurrent psychiatric disorder leads to improvement in the physical and mental health of patients.

In June 2004, a patient focus group discussion in our general practice in Greater London revealed that the detection and treatment of depression was seen as a problem area by patients. In view of this information, and given the evidence for the under-diagnosis of depression in COPD patients, ${ }^{1-3}$ we decided to screen for depression in our population of COPD patients. In doing so we hoped to test the feasibility of screening for depression in COPD patients in primary care, at the same time as identifying and treating any of our COPD patients who were depressed.

\section{Methods}

The study was carried out at the general practice surgery between October and December 2004. The practice has 7300 patients, with a high proportion $(20 \%)$ of elderly patients over the age of 65 years. The sample population consisted of

*Corresponding author: Tel: 07958908619 Fax: 02084201993 E-mail: kamranahmed_786@hotmail.com 
all the patients on the COPD register $(n=69)$. No distinction was made between patients based on spirometry results, as we intended to study patients already diagnosed with COPD at the practice. The one exclusion criteria was a diagnosis of depression requiring current antidepressant treatment $(n=3)$. The remaining 66 patients were sent Beck Depression Inventory (BDI) Short-Form Questionnaires ${ }^{6}$ by post, with an accompanying letter explaining the objectives of the study.

The BDI short-form questionnaire is a 13-item version of the full BDI which identifies various depressive symptoms. A score of 8-15 indicates moderate depression, and a score of 16 and above indicates severe depression. It was decided that patients with a score of 8 or above would be sent appointments for further assessment. Studies have shown that antidepressants and pulmonary rehabilitation are effective treatment modalities for depression in COPD ${ }^{1}$ so it was decided that patients would be offered these as treatment options.

\section{Results}

Of the 66 questionnaires sent out, 44 (66\%) were returned. One patient did not fill in the questionnaire as she felt it did not apply to her. Of the remaining 43 patients, 13 (30\%) were found to have BDI scores of 8 or above. Attempts were made to contact these 13 patients to arrange appointments for review and to discuss treatment options. Of these, 10 were easily contacted by telephone and appointments were arranged. We were unable to contact the remaining three patients despite numerous attempts. In all of the 10 patients who attended for review, the diagnosis of depression was confirmed by clinical interview and they were offered treatment; five chose antidepressants, three chose pulmonary rehabilitation, and two declined treatment (Figure 1).

\section{Discussion}

This study was initiated as a result of a patient focus group discussion in our Greater London practice which highlighted the management of depression as a problem area within the practice. As a consequence, the aim of this study was to screen for depression in our COPD patients. The aims of the study were met; we found a $30 \%$ rate of moderate to severe depression in the COPD patients who responded to our survey. This is a significant proportion, and is in agreement with previous research findings reporting prevalence rates for depression in COPD patients ranging from $20-60 \%,{ }^{1,2}$

There are some methodological limitations to this study. The response rate to the survey was $66 \%$, and so data were not obtained from one third of the COPD patient population. It is possible that this non-responder population had a high proportion of depressed patients who lacked the motivation to complete and return the questionnaire. It is also possible
Figure 1. Flow of patients through trial

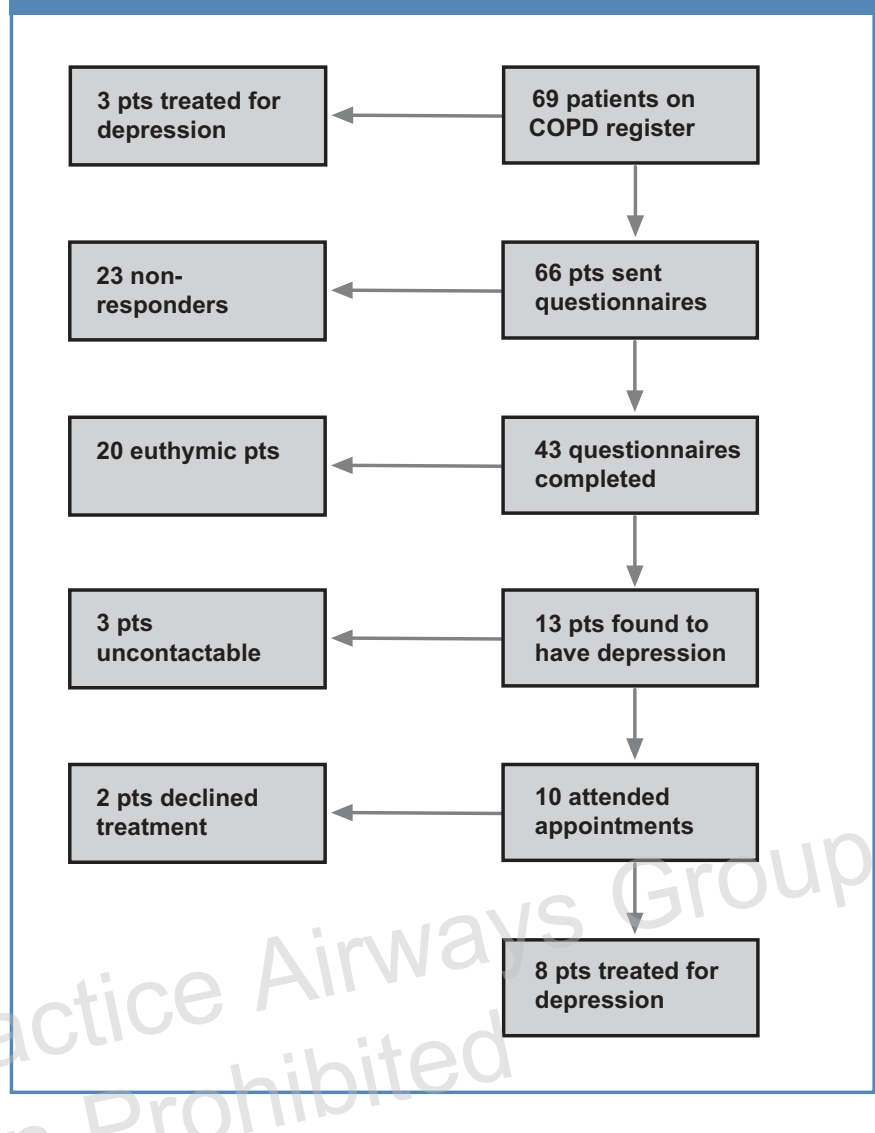

that some patients did not even receive a questionnaire particularly if they had recently moved house or if the questionnaire was lost in the post. We considered alternative methodologies including interviewing all patients with COPD; however, we felt that this would be too time consuming and impractical. We also considered putting up signs or posters in the practice waiting area to raise awareness about depression in COPD and to encourage people to discuss this issue during appointments. However, we felt that this may not be effective in detecting depression in our patient population.

There were two aspects of the study which had ethical considerations. Firstly, it is always a possibility that patients will be concerned when they receive a disease questionnaire by post. The lady who returned the questionnaire uncompleted was unhappy as she did not think she had lung problems since her COPD was so well controlled. Therefore, we took some time to explain the objectives of our study and the meaning of her diagnosis to her. Secondly, there were difficulties in contacting a few patients for follow-up who had reported likely significant depression on their questionnaire reply. This raises issues about the practice's duty of care to these patients, even though we made every effort to contact them and to offer help.

Our study raises certain interesting possibilities; for 
example, could such an approach be useful in identifying depression in other general practices? Could it be used for patients with other chronic illnesses known to harbour a significant proportion of undiagnosed depression? A larger multi-centre study involving a number of general practices would be needed to ascertain the true benefits of such an approach.

For the practice, conducting this study served to raise awareness among doctors and other staff in the practice about the incidence of depression in chronic illnesses such as COPD. Subsequently, this has led to clinicians being more likely to enquire about depressive symptoms during consultations.

\section{Conclusion}

This study illustrates the practical application of a method for screening and treatment of undiagnosed depression in COPD patients in the primary care setting. The distribution by post of the $\mathrm{BDI}$ is relatively cheap, quick and easy. It helped us to identify depression in $30 \%$ of responders, allowing us to treat the illness in 8 out of 13 depressed patients.

\section{Acknow ledgement}

The authors would like to thank Carole Dixey and the rest of the staff at Elliott Hall Medical Centre for all their help during this study.

\section{Conflict of interest declaration}

There are no conflicts of interest to declare

\section{References}

1. Norwood R. Prevalence and impact of depression in chronic obstructive pulmonary disease patients. Curr Opin Pulm Med 2006;12(2):113-17.

2. Roundy K, Cully JA, Stanley MA et al. Are Anxiety and Depression Addressed in Primary Care Patients With Chronic Obstructive Pulmonary Disease? A Chart Review. Prim Care Companion J Clin Psychiatry 2005;7(5):213-18.

3. van Schayck CP, Chavannes $\mathrm{NH}$. Detection of asthma and chronic obstructive pulmonary disease in primary care. Eur Respir J 2003;39Suppl:16s-22s. Review.

4. Curtis JR, Deyo RA, Hudson LD. Pulmonary rehabilitation in chronic respiratory insufficiency. 7. Health-related quality of life among patients with chronic obstructive pulmonary disease. Thorax 1994;49(2):162-70.

5. Mikkelsen RL, Middelboe T, Pisinger C, Stage KB. Anxiety and depression in patients with chronic obstructive pulmonary disease (COPD). A review. Nord J Psychiatry 2004;58(1):65-70.

6. Beck AT, Rial WY, Rickets K. Short form of depression inventory: crossvalidation. Psychol Rep 1974;34(3):1184-6.

\section{Available online at http://w ww.thepcrj.org}

